within 7 days of onset; however, 4/14 lasted 8-21 days and 2/ 14 lasted $>21$ days.

Conclusions Interim data suggest $>91 \%$ of SLE patients did not self-report a flare post-SARS-CoV-2 vaccination; of those that did, most had mild flares. Given most patients reported that their post-vaccine flare was 'typical' of their SLE flares, vaccine side effects alone may not explain these findings. Whether vaccine type or modifying immunosuppressive medications to enhance vaccine efficacy independently predicts SLE flare remains to be determined.

Acknowledgements Dr. Barbhaiya is currently supported by the Rheumatology Research Foundation Investigator Award and the Barbara Volcker Center for Women and Rheumatic Diseases at Hospital for Special Surgery. Dr. Mandl received grant support from Regeneron Pharmaceuticals, and is an Associate Editor at Annals of Internal Medicine. Dr. Siegel is supported by the National Center for Advancing Translational Sciences (NCATS) Grant \# UL1TR02384 of the Clinical and Translational Science Center at Weill Cornell Medical College. The funders had no role in study design, data collection, analysis, decision to publish, or preparation of the manuscript.

\section{COVID-19 PANDEMIC STRESSORS AND PSYCHOLOGICAL DISTRESS SYMPTOMS IN PATIENTS WITH SYSTEMIC LUPUS ERYTHEMATOSUS AND RHEUMATOID ARTHRITIS}

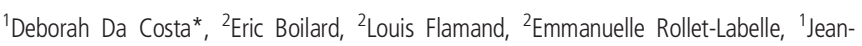
Benoît Deville-Stoetzel, ${ }^{2}$ Louis Bessette, ${ }^{2}$ Karen Adams, ${ }^{2}$ Alexandra Albert, ${ }^{2}$ MarieClaude Audet, ${ }^{2}$ Sonia Lagacé, ${ }^{2}$ Charlotte Grondin, ${ }^{2}$ Philippe Desaulniers, ${ }^{2}$ Paul R Fortin. ${ }^{1}$ Research Institute of the McGill University Health Centre, Quebec, Canada; ${ }^{2}$ Centre de Recherche du CHU de Québec - Université Laval, Québec, Canada

\subsection{6/lupus-2021-lupus21century.69}

Background The coronavirus disease 2019 (COVID-19) global pandemic is a highly stressful event that may contribute to psychological symptoms, particularly in patients with pre-existing chronic conditions. This study examined COVID-19 pandemic related stress experienced by patients with systemic lupus erythematosus (SLE) or rheumatoid arthritis (RA) and its association with symptoms of psychological distress.

Methods An on-line cross-sectional survey study was conducted with 55 SLE (mean age $=54.8, \pm 13.8$ ) and 42 RA (mean age $=64.2, \pm 12.2$ ) patients recruited from a tertiary care centre in Quebec City between May 25, 2021 and June 13, 2021. Participants completed the COVID-19 Stressors Questionnaire adapted by our team for inflammatory arthritis. The Impact of Event Scale-Revised (IES-R) assessed post-traumatic stress symptoms (PTSS) caused by the COVID-19 pandemic. The Patient Health Questionnaire-8 (PHQ-8) and the Generalized Anxiety Disorder-7 (GAD-7) measured symptoms of depression and anxiety, respectively.

Results Among respondents 3/97 had been diagnosed with COVID-19 since the start of the pandemic $(\mathrm{SLE}=2, \mathrm{RA}=1)$. Clinically significant PTSS (IES-R score $\geq 24$ ) due to the COVID-19 pandemic was reported by $13.4 \%$ of participants, with no statistically significant difference between both disease groups (SLE $=16.4 \% ; \mathrm{RA}=9.5 \%$ ). The degree of concern related to COVID-19 stressors were similar in both disease groups (SLE: $\mathrm{M}=10.0 \pm 8.2$; $\mathrm{RA}: \mathrm{M}=8.7 \pm 9.5$ ). As shown in table 1, COVID-19 stressors that were associated with the highest degree of concerns were: having a loved one contract coronavirus (SLE 50.9\%; RA 28.6\%), the possibility of contracting (SLE 45.5\%; RA 35.7\%) or getting sick from coronavirus (SLE 40\%; RA 21.4\%), working in a place with high likelihood of exposure (SLE 30.9\%; RA 21.4\%), and the possibility of their disease worsening or being poorly managed due to changes in medical care (SLE 27.3\%; RA 26.2\%). In patients with SLE, a higher level of concern related to COVID-19 stressors was significantly correlated with greater symptoms of PTSS $(\mathrm{r}=0.46, \mathrm{p}<0.001)$, depression $(\mathrm{r}=$ $0.46, \mathrm{p}<0.001)$ and anxiety $(\mathrm{r}=0.62, \mathrm{p}<0.001)$. In patients with RA, a higher level of concerns related to COVID-19 stressors was significantly correlated with greater symptoms of PTSS ( $\mathrm{r}=0.33, \mathrm{p}-0.036)$, but not with symptoms of depression and anxiety.

Conclusions Stressors related to the COVID-19 pandemic are experienced by an important proportion of patients with SLE and RA and are associated with psychological symptoms, particularly for patients with SLE.

\begin{tabular}{|c|c|c|c|}
\hline \multirow[t]{3}{*}{ COVID-19 Stressors } & All Participants & SLE & RA \\
\hline & $\mathrm{n}=97$ & $\mathrm{n}=55$ & $n=42$ \\
\hline & $\mathrm{n}(\%)$ & $\mathrm{n}(\%)$ & n (\%) \\
\hline Having loved ones who contracts coronavirus & $40(41.2)$ & $28(50.9)$ & $12(28.6)$ \\
\hline Possibility of contracting coronavirus & $40(41.2)$ & $25(45.5)$ & $15(35.7)$ \\
\hline Getting sick from exposure to coronavirus & $31(32)$ & $22(40)$ & $9(21.4)$ \\
\hline Working in a place likely to be exposed to the coronavirus. & $26(26.8)$ & $17(30.9)$ & $9(21.4)$ \\
\hline Possibility of condition worsening or being poorly managed due to changes in medical care & $26(26.8)$ & $15(27.3)$ & $11(26.2)$ \\
\hline Postponement or cancellation of diagnostic and disease monitoring tests & $19(19.6)$ & $10(18.2)$ & $9(21.4)$ \\
\hline Increased responsibilities at home & $19(19.6)$ & $11(20)$ & $8(19.0)$ \\
\hline Difficulty obtaining food, medicine and other essentials & $16(16.5)$ & $9(16.4)$ & $7(16.7)$ \\
\hline Changes in treatments due coronavirus pandemic & $15(15.5)$ & $9(16.4)$ & $6(14.3)$ \\
\hline Postponement or cancellation of medical visits & $15(15.5)$ & $9(16.4)$ & $6(14.3)$ \\
\hline Difficulty obtaining help or social support needed & $12(12.4)$ & $8(14.5)$ & $4(9.5)$ \\
\hline Losing a job or experiencing a drop in income related to the coronavirus pandemic & $10(10.3)$ & $5(9.1)$ & $5(11.9)$ \\
\hline
\end{tabular}

Responses rated on a scale of 0 "not at all" to 4 "extremely" concerned or worried. Responses dichotomized such that a threshold of 2 or higher represent greater concern/worry. 